\title{
AUMENTO DO PODER DISCRIMINATÓRIO DE INDICADORES DE SAÚDE (COEFICIENTES DE MORTALIDADE GERAL E MORTALIDADE INFANTIL)
}

\author{
Affonso Renato Meira * \\ José M. Marlet**
}

RSPU-B/252

MeIRA, A.R. \& MARLET, J.M. - Aumento do poder discriminatório de indicadores de saúde (coeficientes de mortalidade geral e mortalidade infantil). Rev. Saúde públ., S. Paulo, 9:107-13, 1975.

RESUMo: Foi feito estudo demonstrando o aumento do poder discriminatório dos dois coeficientes sanitários mais facilmente calculados em todas as areas do mundo: o coeficiente de mortalidade geral e o coeficiente da mortalidade infantil. A maneira de se obter esse aumento do poder discriminatório consiste em se calcular a diferenca entre estes dois coeficientes de mortalidade. Analisada estatisticamente, usando dados de paises desenvolvidos e em desenvolvimento na mesma época e em épocas diversas, essa diferença demonstrou ser mais discriminatória que qualquer um dos dois isoladamente $e$ quando usada juntamente com um dos dois ou com ambos aumenta significativamente o poder de discriminação.

Unitermos: Niveis de saúde, indicadores. Demografia. Estatística Vital.

\section{N T R O D U ÇA O}

A saúde é um dos valores positivos das coletividades humanas e a melhoria do nível de saúde é um dos objetivos visados por todos os que têm poder decisório na direção desse setor da sociedade.

Todavia, para ser possivel avaliar 0 nível de saúde das populações, é necessário proceder-se a mensurações que permitam comparações.

A medida do nível de saúde é parte fundamental e primária do processo de planificação no setor de saúde. A identificação dos prohlemas, a determinação das prioridades, a formulação de uma política de saúde, a fixação de metas, a designação de recursos humanos e materiais e os fundamentos para o desenvolvimento e avaliação dos programas não têm maior validade $e$ nem merecem maior confiança do que a exatidão que se possa ter alcançado através do diagnóstico da situação (Mejia ", 1967).

()s esforços para se obter um processo para medir o nível de saúde das coletividades dentro de um limite de exatidão razoável intensificaram-se a partir da década de 1950. particularmente após a

* Do Departamento de Medicina Preventiva e Social da Faculdade de Medicina da Fundacão Universitária do $\mathrm{ABC}$ e do Departamento de Saúde das Coletividades da Faculdade de Ciências Médicas de Santos - Caixa Postal 459 - Santos, SP - Brasil

** Do Departamento de Medicina Preventiva da Faculdade de Medicina de Catanduva - Catanduva, SP - Brasil 
MEIRA, A.R. \& MARLET, J.M. - Aumento do poder discriminatório de indicadores de saúde (coeficientes de mortalidade geral e mortalidade infantil). Rev. Saúde públ., S. Paulo, 9:107-13, 1975 .

Assembléia Geral da Organização das $\mathrm{Na}$ ções Unidas, ocorrida em 1952, quando foi solicitado que se dispensasse uma atenção especial às modificações correntes nos niveis de vida. Dentre os diversos itens apontados como aceitáveis internacionalmente para comporem um catálogo dos componentes dos níveis de vida encontra-se a saúde, incluindo as condições demográficas.

Entretanto, um processo que permitisse a mensuração positiva da saúde não pôde ser encontrado pelo fato de que a percepção deste estado está influenciado por diversas variáveis sociais e culturais complexas, difíceis de serem medidas.

A tendência restringiu-se a estimar a perda de saúde, medindo-se a morbidade e a mortalidade das diversas áreas do Globo num dado momento.

A mortalidade geral foi logo considerada como um indicador geral, visto que diz da perda definitiva da condição de saúde. Entretanto, isoladamente, esse coeficiente pouco representa frente à constituição etária das populações e isto levou vários autores a procurarem indicadores mais exatos. Swaroop e Uemura ${ }^{8}$ (1957) propuseram o indicador "mortalidade proporcional de 50 anos e acima" como sugestão para medir o nível de saúde. Esse indicador é hoje, talvez, o indicador geral mais difundido.

Moraes ${ }^{6}$ (1959) elaborou um pouco mais os dados de mortalidade geral propondo para medir os níveis de saúde de coletividades brasileiras uma curva de mortalidade proporcional, quantificada recentemente por Guedes ${ }^{3}$ (1972), atribuindo pesos a cada grupo etário cujo percentual de mortalidade é representado de maneira gráfica na curva.

Outros autores latino-americanos têm se preocupado também com o problema. Entre esses, Gabaldon ${ }^{2}$ (1964) propôs os padrões de mortalidade precoce como base na planificação da ação sanitária e En- derica $^{1}$ (1969) propõe os quartis de mortalidade proporcional, novo indicador de saúde com relação às condições sócio-econômicas.

Nos países em desenvolvimento, as condiçōes de vida expressam-se ainda por altos coeficientes de mortalidade, fazendo com que os indicadores baseados nesta. quando usados corretamente, possam indicar as prioridades entre os problemas a serem solucionados e avaliar os programas estabelecidos. Nos países desenvolvidos, como observou Moriyama ${ }^{7}$ (1964) nota-se que após um declínio do coeficiente de mortalidade como conseqüência do controle das moléstias infectocontagiosas ocorre um estacionamento neste declínio, justificado pelo aumento das doenças crônicas e incidentes. Às vezes, com o "envelhecimento" da população, pode aparecer um aumento no coeficiente de mortalidade geral.

Outro fato verificado é que o coeficiente de mortalidade infantil, com controle das condições ambientais que possam agravar a saúde e que é alcançado com o desenvolvimento sócio-econômico da região, tem uma tendência acentuada para declinar, ficando os casos de morte antes do primeiro ano praticamente restritos a fatores mais ligados ao parto e a acidentes.

Não havendo um indicador que possa medir de maneira realmente satisfatória o nível de saúde de uma região num momento dado, aconselha-se o uso simultâneo de mais de um indicador a fim de se obter um diagnóstico mais exato. Isto porque na mensuração do nível de saúde é necessário atentar para o fato de que se deve selecionar e resumir várias medidas capazes de serem obtidas. Isto não se pode conseguir totalmente com um único indicador (Mejia ${ }^{4}, 1967$ ).

Essas considerações nos levaram ao presente trabalho, no qual é sugerida a melhoria do poder discriminatório dos dois 
MEIRA, A.R. M MARLET, J.M. - Aumento do poder discriminatório de indicadores de saúde (coeficientes de mortalidade geral e mortalidade infantil). Rev. Saúde públ., S. Paulo, $8: 107-13,1975$.

coeficientes de mortalidade mais facilmente obtidos em todas as áreas do mundo: o geral e o infantil.

\section{MATERIAL E METODOS}

Para calcular o que é proposto neste estudo são empregados os coeficientes de mortalidade geral e de mortalidade infantil, ambos na base 1000 usualmente empregada. Para tanto, toma-se o numerador do coeficiente de mortalidade infantil e dele subtrai-se o numerador do coeficiente de mortalidade geral. Esta diferença traduz um valor expresso em termos absolutos.

Para verificar nossa hipótese, de que a diferença dos coeficientes de mortalida- de é mais discriminante do que os coeficientes isolados ou tomados em conjunto. usamos os dados dos mesmos países que Swaroop e Uemura ${ }^{\&}$ (1957) apresentaram em seu estudo. Inicialmente, dados da mesma época, 1949 a 1951, e depois os dados disponíveis mais recentes a respeito dos mesmos países, 1970.

Testamos o poder discriminante da diferença apresentada calculando a distância quadrática $\left(\mathrm{D}^{2}\right)$ de Mahalanobis ${ }^{4}$ (1936) entre os dois grupos de países (desenvolvidos e em desenvolvimento). utilizando os dados contidos nas Tabelas 1 e 2, para cada um dos dois indicadores, assim como para sua aplicação conjunta e sua diferença.

TABELA 1

Coeficientes de mortalidade geral e de mortalidade infantil e as diferencas entre esses coeficientes na base 1.000 em países considerados desenvolvidos em 1949-1951*

\begin{tabular}{|c|c|c|c|}
\hline Paises & Coef. mort. infantil & Coef. mort. geral & Diferença \\
\hline Austrália & 25,0 & 9,6 & 15,4 \\
\hline Canadá & 40,9 & 9,1 & 31,8 \\
\hline Dinamarca & 31,4 & 9,0 & 22,4 \\
\hline Inglaterra e Gales & 30,8 & 12,0 & 18,8 \\
\hline Franca & $\mathbf{5 4 , 5}$ & 13,3 & 41,2 \\
\hline Nova Zelândia & 23,1 & 9,3 & 13,8 \\
\hline Noruega & 27,2 & 8,8 & 18,4 \\
\hline Suécia & 22,0 & 10,0 & 12,0 \\
\hline Sulça & 31,9 & 10,4 & 21,5 \\
\hline Estados Unidos & 29,6 & 9,7 & 19,9 \\
\hline
\end{tabular}

* FONTE: Swaroop e Uemura (1957)

Estudamos a significância estatística dos $\mathrm{D}^{2}$ encontrados com auxílio da distribuição de probabilidades $\mathrm{F}$.

Analisamos, ainda, o aumento do poder discriminante ao associar a diferença proposta aos dois coeficientes de mortalidade, testando este possível aumento pelo método da análise da variância.
Aplicamos o mesmo método para estudar a aplicabilidade dos indicadores para detectar o acréscimo de desenvolvimento de países já desenvolvidos no transcurso de duas décadas, utilizando os dados da Tabela 3.

Marcamos com um asterisco os valores estatísticos considerados como significante ao nível de $5 \%$. 
MEIRA, A.R. \& MARLET, J.M. - Aumento do poder discriminatório de inóicadores de saúde (coeficientes de mortalidade geral e mortalidade infantil). Rev. Saúde públ., S. Paulo, 9:107-13, 1975.

TABELA 2

Coeficientes de mortalidade geral e de mortalidade infantil e as diferencas entre esses coeflcientes na base 1.000 em paises considerados sub-desenvolvidos em 1949-1951*

\begin{tabular}{l|ccr}
\hline \multicolumn{1}{c|}{ Países } & Coef. mort. Infantil & Coef. mort. geral & Diferença \\
\hline Ceilāo & 103,4 & 12,7 & 70,7 \\
Colômbia & 126,0 & 14,2 & 111,8 \\
República Dominicana & 73,2 & 10,1 & 63,1 \\
Egito & 170,5 & 22,5 & 148,0 \\
fndia & 124,8 & 15,4 & 109,4 \\
Malásia & 93,2 & 15,1 & 78,1 \\
México & 100,4 & 17,0 & 83,4 \\
Perú & 104,5 & 12,0 & 92,5 \\
Filípinas & 105,2 & 11,7 & 93,5 \\
Tailândia & 64,5 & 10,3 & $\mathbf{5 4 , 2}$ \\
\end{tabular}

* FONTE: Swaroop e Uemura s (1957)

TA B E L A 3

Coeficientes de mortalidade geral e de mortalidade infantil e as diferenças entre esses coeficientes na base 1.000 em paises considerados desenvolvidos em $1970^{*}$

\begin{tabular}{l|r|r|r}
\hline \multicolumn{1}{c|}{ Paises } & Coef. mort. infantil & Coef. mort. geral & Diferença \\
\hline & 179 & 9,0 & 8,9 \\
Austrálía & 18,8 & 7,3 & $\mathbf{1 1 , 5}$ \\
Canadá & 14,2 & 98 & 4,4 \\
Dinamarca & 18,2 & 11,7 & $\mathbf{5 , 5}$ \\
Inglaterra e Gales & 18,2 & 10,7 & $\mathbf{7 , 5}$ \\
França & 16,7 & 8,8 & $\mathbf{7 . 9}$ \\
Nova Zelândia & 12,7 & 10,0 & $\mathbf{2 , 7}$ \\
Noruega & 11,0 & 9,9 & $\mathbf{1 , 1}$ \\
Suécia & 15,1 & 9,2 & $\mathbf{5 , 9}$ \\
Suiça & 19,8 & 9,4 & $\mathbf{1 0 , 4}$ \\
Estados Unidos & & & \\
\end{tabular}

* FONTE: World Health Statistics Report, 25(9):704-7, 1972

RESULTADOS

Os valores das distâncias quadráticas de Mahalanobis ao comparar países desenvolvidos com países em desenvolvimento, encontram-se na Tabela 4.

Estudamos também o aumento da força discriminante ao associar a diferença a cada um dos coeficientes da mortalidade ou a seu conjunto. Na Tabela 5 apresentamos os valores estatísticos deste estudo.
0 estudo do poder discriminante dos coeficientes estudados e de sua diferença ao serem aplicados a países desenvolvidos, para avaliar o aumento do desenvolvimento alcançado em duas décadas, é apresentado na Tabela 6 .

Neste caso, a significância estatística do aumento de discriminação, ao associarmos a diferença entre os indicadores de mortalidade a cada um deles ou a seu conjunto, é apresentado na Tabela 7 . 
MEIRA, A.R. \& MARLET, J.M. - Aumento do poder discriminatório de indicadores de saúde (coeficientes de mortalidade geral e mortalidade infantil). Rev. Saúde públ., S. Paulo, 9:107-13, 1975 .

\section{T A B E L A 4}

Valores de $D^{*}$ e seu valor estatístico, calculados com os coeficientes de mortalidade infantil $e$ de mortalidade geral e sua diferença para 10 paises desenvolvidos e $10 \mathrm{em}$ desenvolvimento (1949-1951)

\begin{tabular}{|c|c|c|}
\hline Indicadores & $\mathrm{D}^{2}$ & $\mathbf{F}$ \\
\hline Coeficiente de mortalidade infantil & 0,32 & $51,2 *$ \\
\hline Coeficiente de mortalidade geral & 0,01 & $9,8 *$ \\
\hline Diferença entre os anteriores & 0,39 & $56,5^{*}$ \\
\hline Coef. mortalidade geral e infantil & 0,46 & $28,8^{*}$ \\
\hline Coef. mortalidade infantil e diferença & 1,43 & $50,8 *$ \\
\hline Coef. mortalidade geral e diferença & 0,54 & $31,6 \neq$ \\
\hline Coef. mort. geral + mort. infantil + diferença & 5,97 & $65,2 *$ \\
\hline
\end{tabular}

TABELA 5

Valores estatisticos ao acrescentar a diferença entre os coeficientes de mortalidade geral e infantil aos mesmos

\begin{tabular}{lc}
\hline & Indicadores \\
\hline \hline \\
$\begin{array}{l}\text { Coef. mort. infantil } \times \text { (Coef. mert. inf. + diferença) } \\
\text { Coef. mort. geral } \times \text { (Coef. mort. geral + diferença) } \\
\text { (Coef. mort. infantil + geral) } \times \text { (Coef. mort. inf. + geral + diferença) }\end{array}$
\end{tabular}

T A B EL A 6

Valores de $D^{2}$ e seu valor estatístico, calculados com os coeficientes de mortalidade infantil e geral e sua diferença, num grupo de 10 paises considerados desenvolvidos, em $1949 / 1951$ e 1970

\begin{tabular}{|c|c|c|}
\hline & & \\
\hline Indicadores & $\mathrm{D}^{2}$ & $\mathbf{F}$ \\
\hline Coeficiente de mortalidade infantil & 0,07 & $26,2^{*}$ \\
\hline Coeficiente de mortalidade geral & 0,00 & 0,8 \\
\hline Diferença entre os anteriores & 0.08 & $24,9 *$ \\
\hline Coef. de mort. geral e infantil & 0.08 & $11,8 *$ \\
\hline Coef. de mort. infantil e diferença & 0.08 & 11,8 \\
\hline Coef. de mort. geral e diferença & 0,05 & $10,0 *$ \\
\hline Coef. de mort, geral, infantil e diferença & 0,07 & $7,2 *$ \\
\hline
\end{tabular}


MEIRA, A.R. MARLET, J.M. - Aumento do poder discriminatório de indicadores de saúde (coeficlentes de mortalidade geral e mortalidade infantil). Rev. Saúde públ., S. Paulo, 9:107-13, 1975.

TA B E L A 7

Valores estatisticos ao acrescentar a diferença entre os coeficientes de mortalidade geral e infantil a cada um dos mesmos e a seu conjunto

\begin{tabular}{|c|c|}
\hline Ind:cadores & F \\
\hline Coef. mort. infantil $\times$ (Coef. mort. inf. + diferença $)$ & $74,1 *$ \\
\hline Coef. mort. geral $\times$ (Coef. mort. geral + diferença) & $61,8 *$ \\
\hline (Coef. mort. geral + infantil $) \times($ Coef. mort. geral + infantil + diferenca) & $180,0 *$ \\
\hline
\end{tabular}

\section{I S C US S A}

Idealmente não deveria ocorrer mortalidade no primeiro ano de vida, o que levaria o coeficiente de mortalidade infantil a zero. Por outro lado, um maior coeficiente de mortalidade geral associado a um decréscimo da mortalidade infantil, traduz o envelhecimento da população, mostrando um melhor nivel de vida.

A diferença proposta, em conseqüência do exposto anteriormente, quanto mais diminuir, tanto mais traduz boas condições de saúde na área estudada.

Apesar de todos os indicadores empregados neste trabalho serem estatisticamente discriminantes para classificar um país como desenvolvido ou em desenvolvimento, quer quando usados isoladamente, quer quando em conjunto, é possível estabelecer uma ordem hierárquica entre os mesmos, levando em conta sua capacidade discriminante, assim:

- quando empregados isoladamente, o coeficiente geral de mortalidade é o que menos discrimina $\left(D^{2}=0,01\right)$ para diferenciar países desenvolvidos de países em desenvolvimento e não discrimina para mostrar o aumento de desenvolvimento em duas décadas $\left(D^{2}=0,00\right)$;

- a diferença entre os dois coeficientes de mortalidade proposta neste trabalho foi que mostrou ser capaz de maior discriminação nos dois casos;
- as associações de indicadores mostram que a associação dos coeficientes de mortalidade geral e infantil discriminam menos $\left(D^{2}=0,46\right)$ do que cada um deles quando associados à diferença $\left(D^{2}=0,54\right.$ e $D^{2}=1,43$ respectivamente) ;

- a associação que mostrou a máxima capacidade discriminante $\left(D^{2}=5,97\right)$ foi a dos dois coeficientes com a sua diferença, simultaneamente.

As Tabelas 5 e 7 mostram o aumento estatisticamente significante da capacidade discriminante dos dois indicadores ao se associar à diferença proposta, tanto para diferenciar entre países desenvolvidos e em desenvolvimento (Tabela 5), como para avaliar o aumento de desenvolvimento num mesmo país (Tabela 7).

Embora seja praticamente impossivel dizer-se onde acaba o grupo dos países em desenvolvimento e onde começa o dos países desenvolvidos, pois desenvolvimento e subdesenvolvimento são conceitos teóricos e polares, chamamos de desenvolvido todo país que se aproximar do polo "desenvolvimento" e de "em desenvolvimento" quando se aproximar do polo "subdesenvolvido".

A observação das Tabelas 1 e 2 sugere que podem ser classificados como desenvolvidos os países cuja diferença de indicadores é menor que 50 e como em desenvolvimento os países cuja diferença de indicadores é superior a 50 . 
MEIRA, A.R. \& MARLET, J.M. - Aumento do poder discriminatório de indicadores de saúde (coeficientes de mortalidade geral e mortalidade infantil). Rev. Saúde públ., S. Paulo, 9:107-13, 1975.

\section{CONCLUSOES}

1 - A diferença dos coeficientes de mortalidade sugerida mostrou-se capaz de, quando empregada isoladamente:

- discriminar entre países desenvolvidos e em desenvolvimento;

- detectar a mudança de desenvolvimento de países desenvolvidos no transcurso de duas décadas.

2 - Mostrou também que melhora a capacidade discriminante dos coeficientes de mortalidade geral e de mortalidade infantil quando usados isoladamente ou em conjunto:

- para distinguir entre países desenvolvidos e países em desenvolvimento;

- para evidenciar o acréscimo de desenvolvimento de um mesmo país ao longo de duas décadas.

3 - Diferenças de coeficientes de mortalidade, inferior a 50, fazem pensar em país desenvolvido e, superiores a 50 , em país em desenvolvimento.

\section{RSPU-B/252}

MEIRA, A.R. \& MARLET, J.M. - [Increase of discriminatory ability of health indicators (general mortality rate and infantile mortality rate)]. Rev. Saúde públ., S. Paulo, $9: 107-13,1975$.

Summary: A study was made to demonstrate the increase of the discriminative power of two sanitary rates which are most easily calculated in all areas of the world: the general mortality rate and the infantile mortality rate. The manner to obtain this increase of discriminatory power consists in calculating the difference between the infantile mortality rate and the general mortality rate. Statistically analysed and taking into consideration data from developed countries and those undergoing development during the same period, as well as throughout different periods, this difference was more discriminatory than any of the two separately and when used together with one or both, the power of discrimination is increased significantly.

UnIterms: Health levels, indicators. Demography. Vital Statistics.

\section{REFERENCIAS BIBLIOGRAFICAS}

1. ENDERICA, V.R. - Quartiles de mortalidade proporcional - nuovo indicador de salud, com relacion a las condiciones sóclo-econômicas. Rev. Educ. Hig. Med. trop., 26:199-217, 1969 .

2. GABALDON, A. - Padrones de Mortalidad temprana $y$ su uso como base de la planificacion de la accion Sanitária. Bol. Ofic. sanit. panamer., 56:314-23, 1964

3. GUEDES, I. da S. - Contribuicão para o estudo de evolução do nivel de saúde do Estado de São Paulo: análise las Regióes Administrativas (1950-1970). São Paulo, 1972. [Tese - Faculdade de Saúde Pública da Universidade de São Paulo].

4. MAHALANOBIS, P.C. - On the generalized distance in statistics. Proc. Mat. Sci. India, 2:49-55, 1936.

5. MEJIA, V.A. - Medida del nivel de salud em Colombia. [Apresentado na
Conferência Internacional sobre Recursos Humanos para a Saúde e Educação Médica. Maracay, Venezuela, 1967].

6. MORAES, N.L. de A. - Niveis de saúde da coletividade brasileira. Rev. Serv. Saúde públ., Rio de Janeiro, 10:403$97,1959$.

7. MORIYAMA, I.M. - The change in mortality trends in the United States. Washington, D.C., Public Health Service, 1964 (Vital and Health Statistics. Série 3, n.o 1).

8. SWAROOP, P.S.E. \& UEMURA, K. Proportional mortality of 50 years and above. A suggested indicator of the component "Health including demographic conditions" in the measurement of level of living. Bull. Wla. Hlth. Org., 17:439-81, 1957.

Recebido para publicação em 24-02-1975 Aprovado para publicacão em 04-04-1.975 